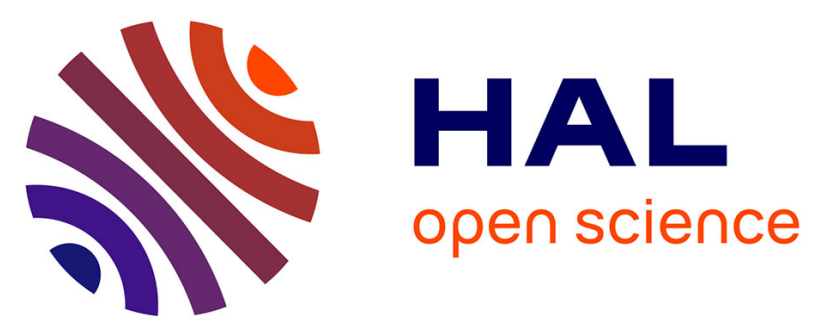

\title{
p53 regulates epithelial-mesenchymal transition through microRNAs targeting ZEB1 and ZEB2
}

Taewan Kim, Angelo Veronese, Flavia Pichiorri, Tae Jin Lee, Young-Jun Jeon, Stefano Volinia, Pascal Pineau, Agnès Marchio, Jeff Palatini, Sung-Suk Suh, et al.

\section{To cite this version:}

Taewan Kim, Angelo Veronese, Flavia Pichiorri, Tae Jin Lee, Young-Jun Jeon, et al.. p53 regulates epithelial-mesenchymal transition through microRNAs targeting ZEB1 and ZEB2. Journal of Experimental Medicine, 2011, 208 (5), pp.875-883. 10.1084/jem.20110235 pasteur-02865376

\section{HAL Id: pasteur-02865376 \\ https://hal-pasteur.archives-ouvertes.fr/pasteur-02865376}

Submitted on 11 Jun 2020

HAL is a multi-disciplinary open access archive for the deposit and dissemination of scientific research documents, whether they are published or not. The documents may come from teaching and research institutions in France or abroad, or from public or private research centers.
L'archive ouverte pluridisciplinaire HAL, est destinée au dépôt et à la diffusion de documents scientifiques de niveau recherche, publiés ou non, émanant des établissements d'enseignement et de recherche français ou étrangers, des laboratoires publics ou privés.

\section{(1)(1) $\$(0)$}

Distributed under a Creative Commons Attribution - NonCommercial - ShareAlikel 4.0 


\section{p53 regulates epithelial-mesenchymal transition through microRNAs targeting ZEB1 and ZEB2}

\author{
Taewan Kim, ${ }^{1,2}$ Angelo Veronese, ${ }^{1}$ Flavia Pichiorri, ${ }^{1}$ Tae Jin Lee, ${ }^{1}$ \\ Young-Jun Jeon, ${ }^{1,2}$ Stefano Volinia, ${ }^{1}$ Pascal Pineau, ${ }^{3}$ Agnès Marchio, ${ }^{3}$ \\ Jeff Palatini, ${ }^{1}$ Sung-Suk Suh, ${ }^{1}$ Hansjuerg Alder, ${ }^{1}$ Chang-Gong Liu, ${ }^{1}$ \\ Anne Dejean, ${ }^{3}$ and Carlo M. Croce ${ }^{1}$ \\ 'Department of Molecular Virology, Immunology and Medical Genetics, Comprehensive Cancer Center, ${ }^{2}$ Molecular, Cellular, \\ and Developmental Biology Program, The Ohio State University, Columbus, $\mathrm{OH} 43210$ \\ ${ }^{3}$ Nuclear Organization and Oncogenesis Unit, Institut National de la Santé et de la Recherche Médicale U579, Institut Pasteur, \\ 75724 Paris Cedex 15, France
}

p53 suppresses tumor progression and metastasis. Epithelial-mesenchymal transition (EMT) is a key process in tumor progression and metastasis. The transcription factors ZEB1 and ZEB2 promote EMT. Here, we show that p53 suppresses EMT by repressing expression of ZEB1 and ZEB2. By profiling 92 primary hepatocellular carcinomas (HCCs) and 9 HCC cell lines, we found that p53 up-regulates microRNAs (miRNAs), including miR-200 and miR-192 family members. The miR-200 family members transactivated by $\mathrm{p} 53$ then repress ZEB1/2 expression. p53-regulated miR-192 family members also repress ZEB2 expression. Inhibition or overexpression of the miRNAs affects p53-regulated EMT by altering ZEB1 and ZEB2 expression. Our findings indicate that p53 can regulate EMT, and that p53regulated miRNAs are critical mediators of p53-regulated EMT.

CORRESPONDENCE

Carlo M. Croce:

carlo.croce@osumc.edu

Abbreviations used: EMT, epithelial-mesenchymal transition; miRNA, microRNA; mut, mutant; p53BS, p53 binding site; qRT-PCR, quantitative real-time PCR; sh, short hairpin RNA; sqRT-PCR, semiquantitative RT-PCR;TSS, transcriptional start site; UTR, untranslated region.
MicroRNAs (miRNAs) are small, noncoding RNAs. They function in various cellular processes by targeting the $3^{\prime}$ untranslated regions (UTRs) of target mRNAs (Bartel, 2004; $\mathrm{He}$ and Hannon, 2004). Differentially expressed miRNAs in tumors inhibit or promote tumor development, progression, and metastasis by regulating oncogenes or tumor suppressor genes (Calin and Croce, 2006).

The tumor suppressor p53 is a transcription factor that induces or represses a large set of genes and miRNAs (Vogelstein et al., 2000; Hermeking, 2007; Chari et al., 2009). Previous studies have shown that deletions or mutations of p53 are frequently found in cancers (Soussi, 2007; Chari et al., 2009), and that p53 is involved in tumor metastasis as well as tumor progression (Lewis et al., 2005; Ridgeway et al., 2006; Chen et al., 2007; Hansen et al., 2007).

Epithelial-mesenchymal transition (EMT) is necessary for many developmental processes,

C.-G. Liu's present address is Dept. of Experimental Therapeutics, Division of Cancer Medicine, University of Texas MD Anderson Cancer Center, Houston, TX, 77030. such as neural tube and mesoderm formation during embryogenesis, and has been highlighted as a key process in tumor invasion, metastasis and tumorigenicity (Yang and Weinberg, 2008; Wellner et al., 2009). EMT, which reprograms transcription of tumor cells, is promoted by transforming growth factors (TGFs), such as TGFB1 (TGF $\beta 1$ ) and TGFB2 (TGF $\beta 2$; Thiery and Sleeman, 2006). Several EMT-activating transcription factors: ZEB1 (dEF1, TCF8, and AREB6), ZEB2 (SIP1), SNAI1 (Snail), SNAI2 (Slug), and TWIST involved in this process have been previously identified (Yang and Weinberg, 2008). Recently, it was reported that the miR-200 family targets ZEB1 and ZEB2 and is significantly down-regulated in TGF $\beta$-induced mesenchymal cells and cancer cells with mesenchymal characteristics (Gregory et al., 2008; Park et al., 2008). Here, we have expanded the knowledge about EMT and p53. We show first

(0) $2011 \mathrm{Kim}$ et al. This article is distributed under the terms of an AttributionNoncommercial-Share Alike-No Mirror Sites license for the first six months after the publication date (see http://www.rupress.org/terms). After six months it is available under a Creative Commons License (Attribution-Noncommercial-Share Alike 3.0 Unported license, as described at http://creativecommons.org/licenses/ by-nc-sa/3.0/) 
that p53 prevents EMT by repressing ZEB1 and ZEB2 expression and, second, that p53-regulated miR-200 and miR-192 family members are involved in p53-modulated EMT.

\section{RESULTS AND DISCUSSION}

p53 suppresses epithelial-mesenchymal transition

EMT-generated mesenchymal cells are characterized by downregulated $\mathrm{CDH} 1$ expression and up-regulated $\mathrm{CDH} 2$ and/or
Vimentin (VIM) expression (Yang and Weinberg, 2008). EMT results in increased cell invasion and migration and is frequently accompanied by disseminated cell growth and changes in cellular morphology (i.e., cells become thin and elongated.). We found that p53 deletion causes significant changes in cell morphology and dissemination corresponding to EMT characteristics (Fig. 1 A). In addition, p53 deletion or knock-down resulted in enhanced cell invasion (Fig. 1 B) and migration (Fig. 1, C and D).
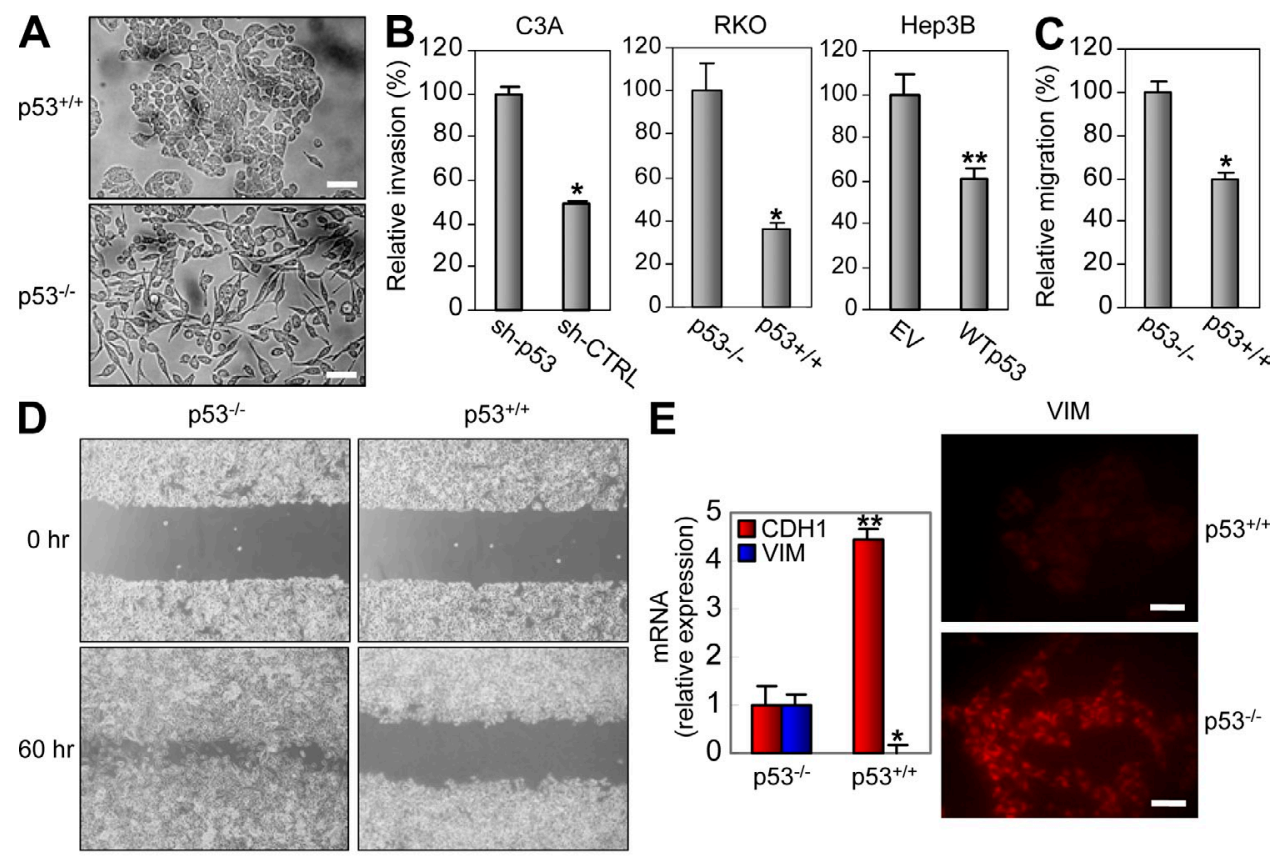

$E$

VIM
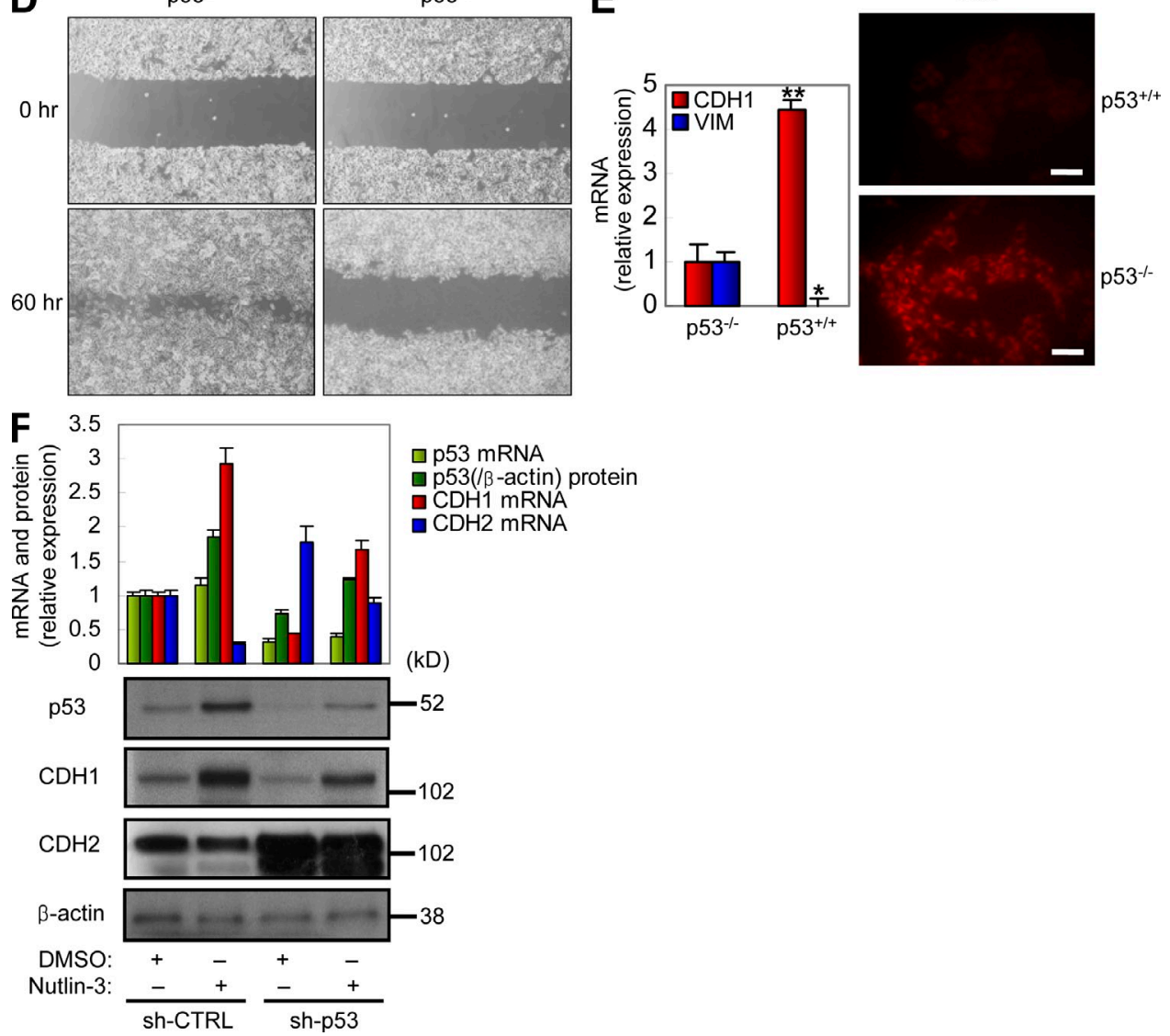

Figure 1. p53 inhibition induces EMT resulting in increased cell invasion and migration. (A) Phase-contrast view of $R K 0\left(p 53^{+/+}\right.$and $p 53^{-1-}$ )

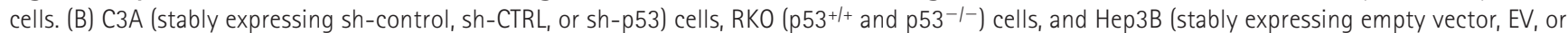
WT p53 expression vector [WTp53]) cells were subjected to in vitro invasion assay for $48 \mathrm{~h}$. (C) RKO (p53+/+ and p53 ${ }^{-1-}$ ) cells were subjected to in vitro migration assay for $24 \mathrm{~h}$. (D) Wound-healing assay using RKO ( $553^{+/+}$and $p 53^{-l^{-}}$) cells that were plated and disrupted with a $200-\mu \mathrm{l}$ tip. $60 \mathrm{~h}$ after disruption, the cell layer was photographed. (E) Relative expression levels of CDH1 and VIM mRNA (left) and VIM protein (right) in RKO (p53+l+ and p53-1-) cells. (F) Relative expression of $\mathrm{CDH} 1$ and $\mathrm{CDH} 2$ mRNA (top) and protein (bottom) in C3A cells stably expressing sh-CTRL or sh-p53 and treated with DMSO or $10 \mu \mathrm{M}$ nutlin-3 for $24 \mathrm{~h}$. (top) Densitometry values of p53 protein levels were normalized with those of $\beta$-actin protein levels. $(A$ and $D) A$ representative experiment out of three independent experiments. $(B, C, E$ and $F)$ Data are mean $\pm S E M$ of three independent experiments and each measured in triplicate $\left({ }^{*}, \mathrm{P}<0.05 ;{ }^{*}, \mathrm{P} \leq 0.01\right)$. Bars, $50 \mu \mathrm{m}$. 
To verify that the changes in cell morphology and dissemination are caused by EMT, expression levels of epithelial and mesenchymal markers were compared with p53 expression level. The epithelial marker (CDH1) was consistently repressed, whereas mesenchymal markers (CDH2 orVIM) were induced by $\mathrm{p} 53$ inhibition (Fig. 1, E and F). In addition, p53 activation using nutlin-3 (Vassilev et al., 2004) also induced $\mathrm{CDH} 1$, but reduced $\mathrm{CDH} 2$ expression levels (Fig. $1 \mathrm{~F}$ ). These observations indicate that p53 inhibits EMT and causes decreased tumor invasion and migration.

\section{p53 represses ZEB1 and ZEB2 expression through miRNAs}

To identify EMT regulators that are involved in the p53regulated EMT, we tested expression levels of the following known EMT activators: SNAI1, SNAI2, TGFB1, TGFB2, TWIST, ZEB1, and ZEB2. The expression levels of EMT activators such as SNAI1, SNAI2,TGFB1, and TGFB2 were not induced by p53 inhibition (Fig. S1, A-D). TWIST was not detected at either the mRNA or protein level (unpublished data). However, expression levels of ZEB1 and ZEB2 were significantly repressed by p53 (Fig. 2,A and B and Fig. S2 A), suggesting a potent role of ZEB1 and ZEB2 in p53-regulated EMT.

First, we attempted to determine whether ZEB1 and ZEB2 are involved in p53-mediated EMT regulation. To assess this hypothesis, ZEB1 and ZEB2 were knocked down in p53 $3^{-1-}$ RKO cells by stable expression of short hairpin RNA (sh)-ZEB1 and sh-ZEB2. In p53-/- RKO cells, the simultaneous repression of ZEB1 and ZEB2 significantly inhibited mesenchymal cell morphology and dissemination (Fig. 2 C). However, when ZEB1 or ZEB2 repression occurred separately, that repression did not inhibit the mesenchymal characteristics significantly (Fig. S2, B and C). Furthermore, simultaneous ZEB1 and ZEB2 repression decreased cell invasive and migratory capacity, which was increased by p53 knock-down or knock-out (Fig. 2, D and E). These results suggest that, together, ZEB1 and ZEB2 play a key role in p53-mediated EMT regulation.

Next, we examined the mechanism by which $\mathrm{p} 53$ represses ZEB1 and ZEB2 expression. ZEB1 protein levels were significantly induced by p53 inhibition (Fig. 2, A and B). At the same time, ZEB1 mRNA levels were also significantly upregulated by p53 deletion or knock-down (Fig. S3 A). These findings suggest that ZEB1 is repressed by p53 at either the transcriptional or mRNA level. To examine whether ZEB1 is repressed by $\mathrm{p} 53$ at the transcriptional level, luciferase reporters containing the $5^{\prime}$ promoter regions of ZEB1 were expressed in p53-expressing C3A cells (C3A-sh-CTRL) and p53 knocked-down C3A cells (C3A-sh-p53). Unexpectedly, we found that luciferase activities were higher in p53-expressing cells than in the p53 knocked-down cells (Fig. S3 B). This result indicates that p53 may activate rather than repress the transcription of ZEB1 mRNA. However, p53 represses ZEB1 protein levels as we showed (Fig. 2,A and B). Thus, these results suggest that ZEB1 is repressed by p53 at the mRNA level.

ZEB2 mRNA levels were not significantly altered by p53 (Fig. S3 A), although ZEB2 protein levels were dramatically
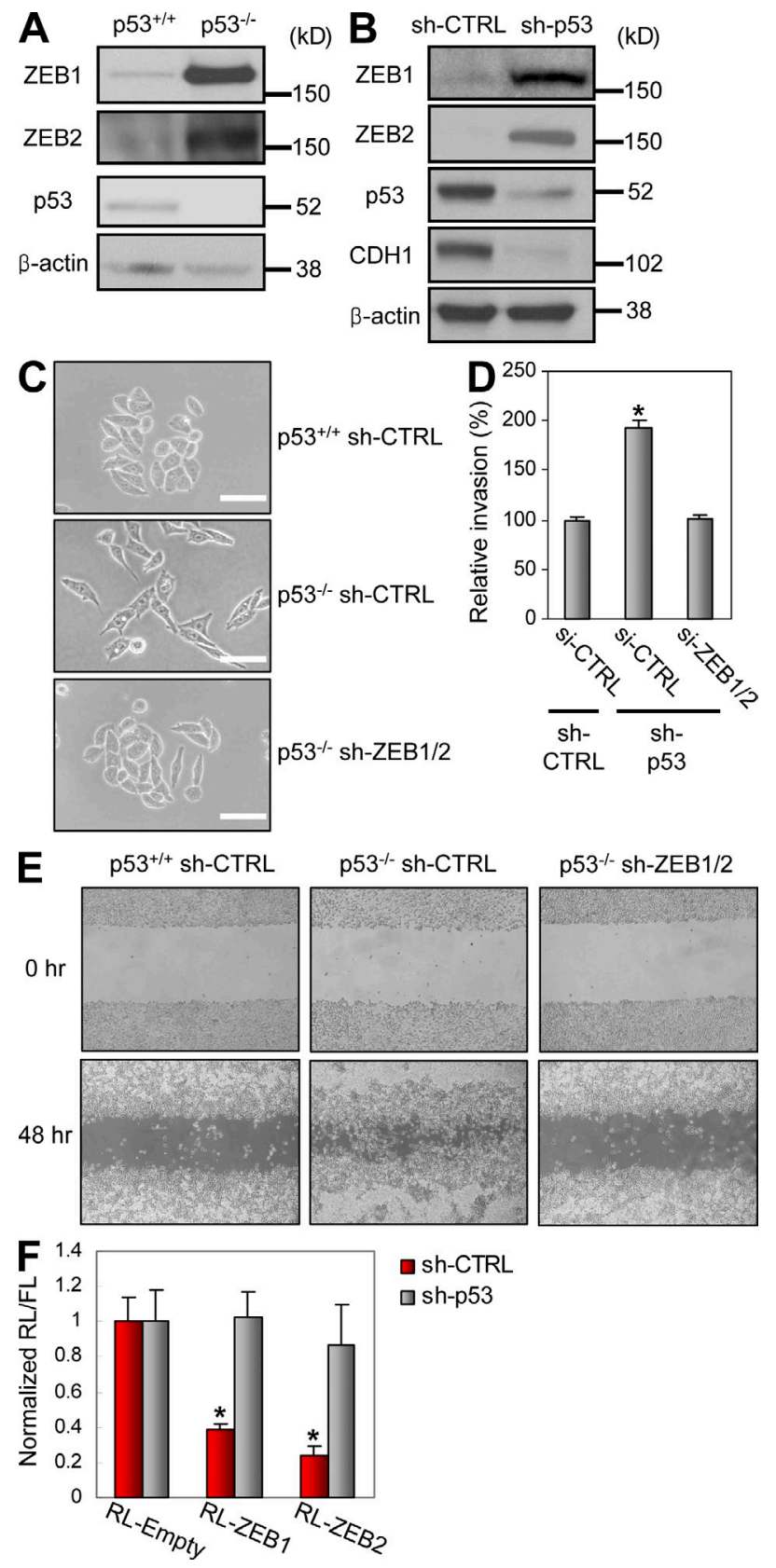

Figure 2. p53 inhibits EMT through ZEB1 and ZEB2 repression in a 3'UTR-dependent manner. (A) Protein levels of ZEB1, ZEB2, and p53 in RKO ( $p 53^{+++}$and $p 53^{-1-}$ ) cells. (B) Protein levels of ZEB1, ZEB2, p53, and $\mathrm{CDH} 1$ in C3A-sh-CTRL and C3A-sh-p53 cells. (C) Phase contrast view of RKO ( $p 53^{+/+}$and $p 53^{-1-}$ ) cells stably expressing sh-CTRL or shZEB1/2. (D) In vitro invasion assay using C3A-sh-CTRL and C3A-sh-p53 cells transfected with si-CTRL or si-ZEB1/2. (E) Wound-healing assay using RKO $\left(\mathrm{p} 53^{+/+}\right.$and $\mathrm{p} 53^{-/-}$) cells stably expressing sh-CTRL or sh$Z E B 1 / 2$. A representative experiment out of three independent experiments. (F) Luciferase assay of ZEB1 (RL-ZEB1) or ZEB2 (RL-ZEB2) 3'UTR in C3A-sh-CTRL and C3A-sh-p53 cells. RL, Renilla luciferase; FL, Firefly luciferase. (A-C) A representative experiment out of two independent experiments. ( $D$ and F) Data are mean \pm SEM of three independent experiments and each is measured in triplicate $\left({ }^{*}, P<0.05 ;{ }^{*}, P \leq 0.01\right)$. Bars, $50 \mu \mathrm{m}$. 
repressed by $\mathrm{p} 53$. These findings suggest that ZEB2 is repressed by $\mathrm{p} 53$ at the posttranscriptional level such as the translational or protein (posttranslational) level. To examine whether p53 regulates ZEB2 expression at the protein level, ZEB2 expression vectors lacking the 3' UTR were introduced into $\mathrm{p} 53^{-/-}$cells with either the empty or $\mathrm{p} 53$ expression vectors. However, p53 did not reduce exogenous ZEB2 protein levels (Fig. S3 C). These results indicate that the ZEB2 expression is regulated by p53 at the translational level and that the 3' UTR of ZEB2 mRNA may be implicated in the mechanism of $\mathrm{p} 53$-repressed ZEB2 expression.

All of these findings indicate that the expression of ZEB1 and ZEB2 is regulated at the mRNA or translational level. In addition, the findings are consistent with the knowledge that miRNAs target mRNAs, resulting in degradation or translational inhibition of the mRNAs (Bartel, 2004;
He and Hannon, 2004). To investigate if ZEB1 and ZEB2 mRNAs are repressed by $\mathrm{p} 53$-dependent miRNAs, luciferase reporters inserted with the 3' UTR of ZEB1 or ZEB2 (Fig. S4 A) were expressed in cells stably expressing sh-CTRL or sh-p53. This resulted in decreased luciferase activities in p53-expressing cells (sh-CTRL) but not in p53 knockeddown cells (sh-p53; Fig. 2 F), indicating that miRNAs may be involved in p53-repressed ZEB1 and ZEB2 expression.

\section{miR-200 family members are regulated by $\mathrm{p} 53$}

To identify miRNAs that are regulated by $\mathrm{p} 53$, we used miRNA microarray analysis (Liu et al., 2004). We analyzed 92 primary hepatocellular carcinomas (HCCs) and 9 HCC cell lines that were classified by p53 status (Materials and methods). The microarray data from the primary HCC samples (Table S1) were compared with the data from the HCC cell
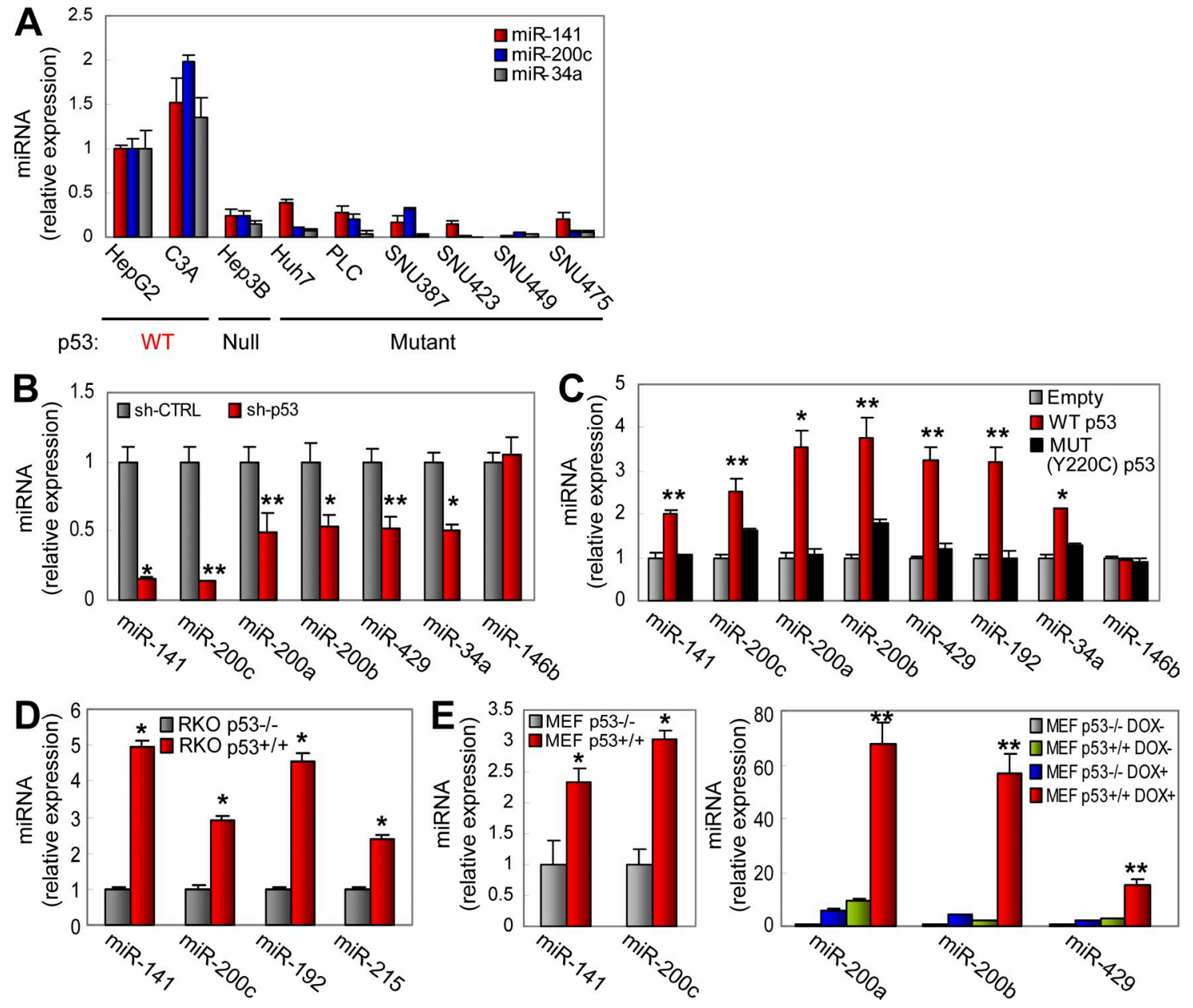

Figure 3. miR-200 and miR-192 family members are regulated by p53. (A) Expression levels of miR-141, miR-200c, and miR-34a in nine HCC cell lines harboring WT p53, p53 deletion (Null), or mutant p53 (Mutant). (B) Relative expression of miR-200 family members, miR-34a (positive control), and miR-146b (negative control) in C3A-sh-CTRL and C3A-sh-p53 cells. (C) Relative expression levels of miR-200 family members, miR-192, miR-34a (positive control), and miR-146b (negative control) in Hep3B cells stably expressing WT p53, mutant (MUT) p53, or empty vectors. (D) Expression levels of miR-141,

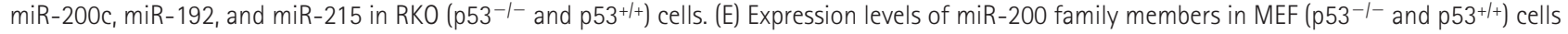
with (right) or without (left) doxorubicin $(200 \mathrm{ng} / \mathrm{ml})$ treatment for $24 \mathrm{~h}$. (A-E) Data are mean \pm SEM of three independent experiments and each is measured in triplicate $\left(* *, P<0.05 ;{ }^{*}, \mathrm{P} \leq 0.01\right)$. 
lines (Table S2). From these comparisons, we identified nine miRNAs (miR-141, miR-192, miR-193b, miR-194, miR200b, miR-200c, miR-215, miR-224, and miR-34a) as p53 up-regulated miRNAs.

Although previously published data have shown that miR-34a and miR-192 family members (miR-192 and miR215) can be regulated by p53 (Chang et al., 2007; He et al., 2007; Raver-Shapira et al., 2007; Braun et al., 2008; Georges et al., 2008; Pichiorri et al., 2010), the regulation of miR-200 family by 53 in tumor samples has never been investigated before. By examining the expression levels of miR-141 and
miR-200c, we found that the miRNAs are highly expressed in HCC cell lines harboring WT p53 (Fig. 3 A). In addition, miR-200 and miR-192 family members were repressed by p53 inhibition and/or induced by p53 activation in various cell types (Fig. 3, B-E; and Fig. S5 A).

To determine whether $\mathrm{p} 53$ regulates miR-200 family members at the transcriptional level, various regions of miR-200 family members were amplified by semiquantitative RT-PCR (sqRT-PCR). These include portions of the primary miRNAs (primary transcripts) that are not located in the section containing precursor miRNAs (Fig. 4 A). Expression levels of these
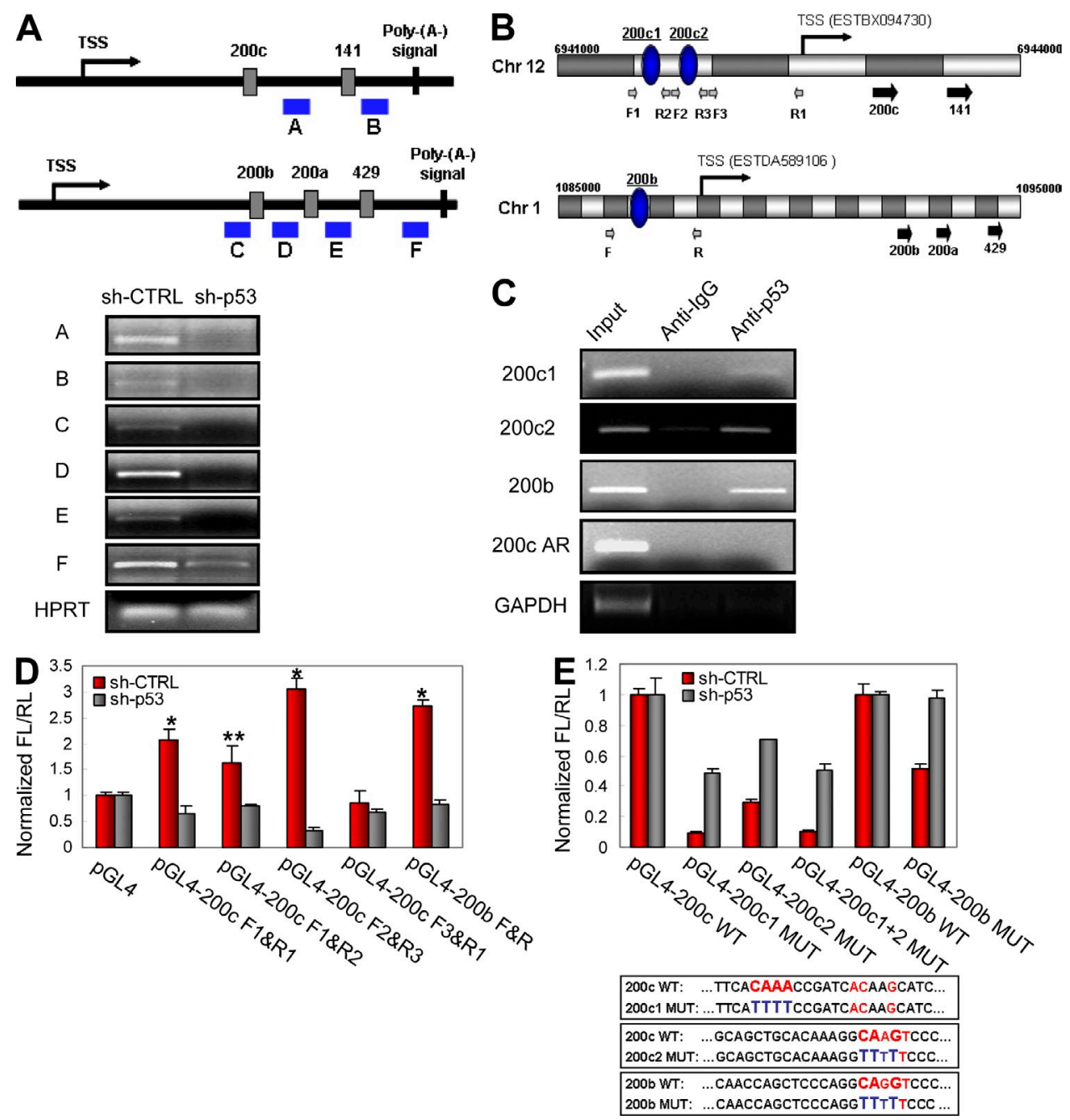

Figure 4. miR-200 family members are directly regulated by $\mathrm{p} 53$ at the transcriptional level. (A) sqRT-PCR of miR-200 family clusters. The regions (A-F) amplified by sqRT-PCR are indicated with blue-colored bars in a schematic (top). (B) Schematic view of miRNA-200 family clusters and chromosomal locations of TSS and putative p53BS (blue ovals: 200c1, 200c2, and 200b). Gray and white fragments represent 500 bp in length. Small gray arrows indicate primers ( $F$, forward; $R$, reverse) used for the constructs of luciferase assay (D). Large black arrows indicate locations of precursor miRNAs. (C) ChIP analysis using C3A cells. 200c1 and 200c2 designate p53BSs of miR-200c-141 cluster and 200b designates p53BS of miR-200b-429 cluster. 200c AR designates a region adjacent ( 200 bp from 200c2) to p53BS of miR-200c-141 cluster. 200c AR and GAPDH are used as negative controls. Input represents $1 \%$ of the total chromatin used in this analysis. (D) Luciferase assay of $5^{\prime}$ promoters of miR-200 family clusters in C3A-sh-CTRL and C3Ash-p53 cells. The regions inserted into luciferase reporters are depicted in B. RL, Renilla luciferase; FL, Firefly luciferase. (E, top) Luciferase assay of $5^{\prime}$ promoter with WT or mutant (MUT) p53BSs of miR-200 family clusters in C3A-sh-CTRL and C3A-sh-p53 cells. (bottom) Sequence information on p53BSs mutations. ( $A$ and $C$ ) A representative experiment out of two independent experiments. ( $D$ and $E$ ) Data are mean $\pm S E M$ of three independent experiments and each is measured in triplicate $\left({ }^{*}, \mathrm{P}<0.05{ }^{*}{ }^{*}, \mathrm{P} \leq 0.01\right)$. 
primary miRNA regions in miR-200c-141 (Fig. 4 A, A and B) and miR-200b-429 (Fig. 4 A, C-F) clusters were significantly reduced by $\mathrm{p} 53$ inhibition, suggesting that $\mathrm{p} 53$ regulates miR200 family members at the transcriptional level.

It has been reported that the clusters of miR-200c-141 and miR-200b-429 are encoded by single polycistronic transcripts, and putative transcriptional start sites (TSSs) of the transcripts have also been identified (Bracken et al., 2008; Burk et al., 2008). Here, we used MatInspector to analyze putative p53 binding sites (p53BSs) in $5^{\prime}$ promoter regions of the transcripts (Cartharius et al., 2005). Using the prediction tool, we found two putative p53BSs (200c1 and 200c2) and one putative p53BS (200b) in the $5^{\prime}$ promoter regions of the miR-200c-141 and miR-200b-429 clusters (Fig. 4 B).

To verify the prediction for the p53BSs, we performed chromatin immunoprecipitation (ChIP) assay in C3A cells using p53-specific antibody. The result of ChIP assay shows the direct binding of p53 on the p53BSs (Fig. 4 C). Luciferase activities driven by the $5^{\prime}$ promoter regions (Fig. $4 \mathrm{~B}$ ) of miR$200 \mathrm{c}-141$ and miR-200b-429 clusters were induced in p53expressing (C3A-sh-CTRL) cells, but not in p53-knock-down (C3A-sh-p53) cells (Fig. 4 D, lane 2 and 6). In addition, luciferase reporters including each p53BS (Fig. 4 B) of miR-200c141 cluster also showed higher activities in C3A-sh-CTRL than C3A-sh-p53 cells (Fig. 4 D, lane 3 and 4). To further confirm the function of the p53BSs, they were mutated as indicated (Fig. 4 E, bottom) and luciferase reporters containing the $5^{\prime}$ promoter region mutations were expressed in C3Ash-CTRL and C3A-sh-p53 cells. To compare the relative reduction of luciferase activity in the cells, luciferase activities of the mutated luciferase reporters were normalized with those of WT luciferase reporters. As a result, the mutations in the p53BSs resulted in more significantly reduced luciferase activities in C3A-sh-CTRL cells than in C3A-sh-p53 cells (Fig. 4 E, top). Collectively, these results indicate that p53 directly activates transcription of miR-200 family members.

\section{p53-regulated miR-200-192 family is involved in p53-mediated EMT}

It has been reported that miR-200 family members target ZEB1 and ZEB2 mRNAs (Burk et al., 2008; Gregory et al., 2008; Park et al., 2008), and that miR-192 targets ZEB2 mRNA (Kato et al., 2007). We also observed that miR-200 family members repress ZEB1 and ZEB2 expression. This resulted in increased CDH1 and decreased VIM expression (Fig. S5, B and C). In addition, we observed that miR-192 represses ZEB2, but not ZEB1, expression (Fig. S5 D, left).We next examined the role of miR-200 and miR-192 family members in p53-mediated EMT regulation. By overexpressing miR-141, $\mathrm{miR}-200 \mathrm{c}$, and/or miR-192, mesenchymal characteristics (e.g., changes to cell morphology and enhanced dissemination, invasion, and migration) caused by p53 deletion were significantly inhibited (Fig. 5, A and B). These results suggest that the miR-200 family members and miR-192 are involved in p53mediated EMT regulation and that miR-192 targeting ZEB2 but not ZEB1 also has a role in p53-regulated EMT.
Next, we examined the function of endogenous miR-200 and miR-192 family members using luciferase reporters. Inhibition of miR-141 or miR-200c induced luciferase activities of ZEB1 and ZEB2 3' UTRs (Fig. 5 C). Inhibition of miR-192 or miR-215 induced luciferase activity of ZEB2 3' UTR (Fig. 5 D) and miR-192 inhibition induced ZEB2 protein levels (Fig. S5 D, right). To further test the function of the miR-200 family in p53-repressed ZEB1 and ZEB2 expression, C3A-sh-CTRL and C3A-sh-p53 cells were transfected with luciferase reporters containing the ZEB1 or ZEB2 3' UTR with mutations in miR-200 family binding sites (Fig. S4 B). The mutations in the miR-200 family binding sites resulted in more significant induction of luciferase activities in p53expressing cells than in p53 knocked-down cells (Fig. 5 E). These results indicate that miR-200 and miR-192 family members have a substantial function in p53-mediated ZEB1/2 repression. In addition, miR-141 and miR-200c expression levels correlated to the CDH1 expression level in primary HCC samples, supporting the role of endogenous miR-200 family in EMT (Fig. S5 E). Collectively, our results demonstrate that the miR-200 and miR-192 family members regulated by p53 play a critical role in p53-repressed EMT.

In summary, we show that p53-regulated miRNAs prevent EMT by repressing ZEB1 and ZEB2 expression. Our results complement a recent study that shows p53-regulated EMT through miR-200c-ZEB1 (Chang et al., 2011). By sequencing and profiling $92 \mathrm{HCC}$ samples, we further revealed that miR200 and miR-192 family members are p53-regulated miRNAs and regulate ZEB1 and ZEB2 protein expression at the posttranscriptional level. In addition, our results indicate that both ZEB1 and ZEB2, which are targeted by a cohort of p53-regulated miRNAs, are involved in p53-modulated EMT. Many studies have shown that both p53 and EMT have a critical role in tumor invasion, metastasis, and tumorigenicity (Lewis et al., 2005; Ridgeway et al., 2006; Chen et al., 2007; Hansen et al., 2007; Yang and Weinberg, 2008; Wellner et al., 2009). Thus, our observations may provide new insights into the role of p53 and miRNAs in tumor invasion, metastasis, and tumorigenicity.

\section{MATERIALS AND METHODS}

Patients and primary HCC samples. This study was approved by an Institutional Human Research Review Board (RBM 2005-019). We followed established protocols regarding informed consent and anonymity. Our research team collected and analyzed tissue from 92 HCC patients. Tissue specimens were obtained retrospectively under strict anonymity from historical collections established in surgery or pathology units of hospitals. All samples were fresh-frozen in liquid nitrogen after surgery and kept at $-80^{\circ} \mathrm{C}$. Frozen tissue samples were homogenized using the Tissue Ruptor (QIAGEN) before RNA extraction. Total RNA was extracted using TriReagent (MRC) in accordance with the manufacturer's instructions. To determine TP53 (p53) status of the primary HCC samples, PCR products of TP53 exons 4-11 from genomic DNA were directly sequenced because $>95 \%$ of TP53 mutations in HCC occur in these exons. Of 92 samples, 62 harbored WT TP53. However, TP53 was mutated or deleted in 30 of the samples.

Migration and invasion assays. Cell migration and invasion assays were performed with Cell Migration Assay kit (Millipore) and Cell Invasion Assay 


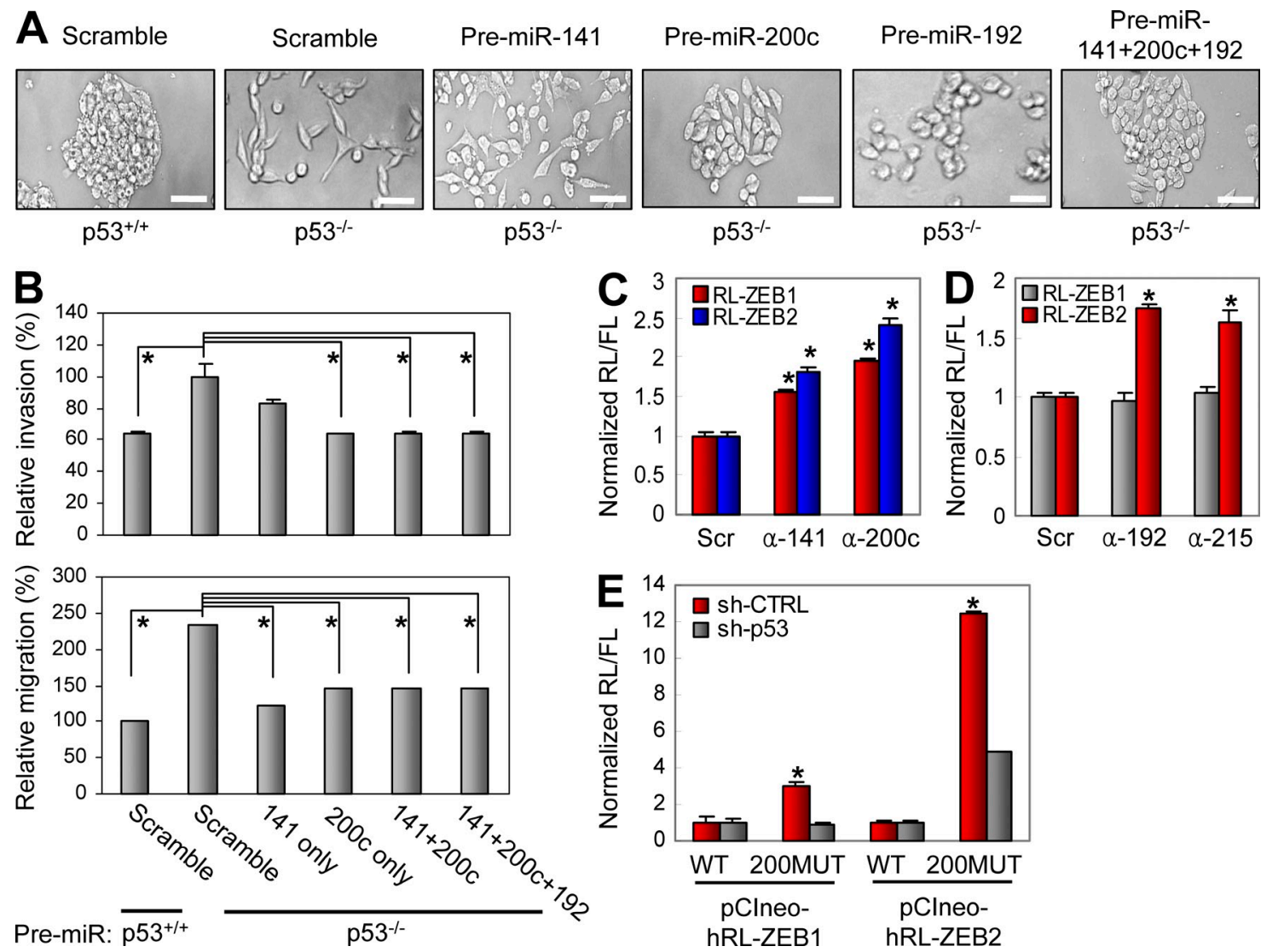

Figure 5. miR-200 and miR-192 family members regulate p53-mediated EMT by targeting ZEB1 and ZEB2. (A) p53+/+ and p53-1- RKO cells were transfected twice a week for 2 wk with indicated premiRs ( $50 \mathrm{nM}$ ). Cells were then photographed. A representative experiment out of three independent experiments is shown. (B) RKO ( $553^{+/+}$and $\mathrm{p5}^{-{ }^{--}}$) cells transfected with indicated premiRs were subjected to in vitro invasion (top) and migration (bottom) assays. (C) C3A cells were transfected with luciferase constructs driven by 3'UTRs of ZEB1 or ZEB2 (RL-ZEB1, RL-ZEB2), together with scrambled miRNA inhibitor (Scr) or inhibitors of miR-141 ( $\alpha-141)$ or miR-200c $(\alpha-200 c)$. RL, Renilla luciferase; FL, Firefly luciferase. (D) RKO (p53+l+) cells were transfected with luciferase constructs driven by 3'UTRs of ZEB1 or ZEB2 (RL-ZEB1, RL-ZEB2), together with scrambled miRNA inhibitor (Scr) or inhibitors of miR-192 ( $\alpha$-192) or miR-215 ( $\alpha-215)$. (E) Luciferase assay using C3A-sh-CTRL and C3A-sh-p53 cells transfected with pClneo-hRL constructs containing ZEB1 or ZEB2 3'UTR with (200MUT) or without (WT) mutations in miR-200 family binding sites. (B-E) Data are mean \pm SEM of three independent experiments and each is measured in triplicate $\left({ }^{*}, \mathrm{P}<0.05 i^{*}, \mathrm{P} \leq 0.01\right)$. Bars, $50 \mu \mathrm{m}$.

kit (Millipore). Both procedures were carried out in accordance with the manufacturer's instructions. In brief, cells were starved in serum-free medium for $24 \mathrm{~h}$, and then plated in the chamber $(8 \mu \mathrm{m}$ pole size) containing uncoated polycarbonate membrane (migration assay) or coated with a thin layer of polymerized collagen (invasion assay). The chamber was incubated in a well containing $10 \%$ serum-containing medium for $24 \mathrm{~h}$ (migration assay) or $48 \mathrm{~h}$ (invasion assay). The cells on the bottom of the chamber were stained and dislodged. We then measured those cells in accordance with the manufacturer's instructions.

Wound-healing assay. When cultured cells reached $90 \%$ confluence, they were starved for $24 \mathrm{~h}$ by incubation in serum-free medium. After the $24 \mathrm{~h}$ starvation, cells were scratched with a pipette tip and cultured in medium containing 10\% FBS. Recovery of the disruption was observed for up to $60 \mathrm{~h}$.

Cells, reagents, and antibiotics. HCC cell lines (HepG2, C3A, Hep3B, Huh7, PLC/PRF/5, SNU-387, SNU-423, SNU-449, and SNU-475) were cultured as recommended by the American Type Culture Collection. RKO $\left(\mathrm{p} 53^{-/-}\right.$and $\mathrm{p} 53^{+/+}$) cells were gifts from B. Vogelstein (Johns Hopkins University, Baltimore, MD). The cells were maintained in Eagle's Minimum Essential Medium with 10\% FBS (Sigma-Aldrich). Mouse embryonic fibroblasts (MEFs) were gifts from G. Leone (The Ohio State University, Columbus,
$\mathrm{OH})$. The cells were cultured in DME with 10\% FBS. RKO cells stably transfected with sh-ZEB1 and sh-ZEB2 were generated by lentiviral sh-ZEB1 transfection and puromycin selection, followed by ZEB2 sh-RNA constructs transfection and hygromycin b selection. Control RKO (p53-/- sh-CTRL and $\mathrm{p} 53^{+/+}$sh-CTRL) cells were also generated by the same procedures using lentiviral sh-CTRL and control sh-RNA constructs. 5-fluorouracil (5-FU) and Doxorubicin were purchased from Sigma-Aldrich. Nutlin-3a was obtained from Cayman Chemical Company. G418 was from purchased from Roche, and puromycin was purchased from Santa Cruz Biotechnology, Inc. Hygromycin b was obtained from Sigma-Aldrich.

Plasmids, oligonucleotides, and transfections. The p53 sh-RNA plasmid and lentiviral particles of sh-ZEB1, sh-ZEB2, and sh-CTRL were purchased from Santa Cruz Biotechnology, Inc. ZEB2 and control sh-RNA constructs were purchased from SA Biosciences. The Zeb2-HA expression plasmid was a gift from A.H. Lund (University of Copenhagen, Copenhagen, Denmark). Precursor miRNAs (Pre-miR) were obtained from Ambion, and LNA-miRNA inhibitors (LNA-miR) were obtained from Exiqon. p53 expression plasmids (WT or mutant) were cloned from the cDNA of HepG2 (WT), Huh7 (Y220C), or SNU-449 (A161T) into pcDNA3.1 (Invitrogen) using BamH1 and EcoRI. For promoter assays, the $5^{\prime}$ promoter regions indicated in the figures were amplified by PCR. The PCR products amplified 
from human genomic DNA containing the 5' promoter regions of the miR-200c-141 cluster were inserted into pGL4-luc2P vectors (Promega) using KpnI and HindIII or XhoI and HindIII. And the PCR products from human genomic DNA containing the $5^{\prime}$ promoter region of the miR-200b429 cluster was inserted into pGL4-luc2P vectors using Kpn1 and NheI.The plasmids containing mutations in p53BSs in pGL4-200c F1\&R1 (p53BS200c MUT) and pGL4-200b F\&R (p53BS-200b MUT) were generated using QuikChange Site-Directed Mutagenesis kit (Stratagene) or QuikChange Multi Site-Directed Mutagenesis kit (Stratagene). For the 3'UTR assay, the $3^{\prime} \mathrm{UTR}$ regions for the mRNAs of interest were PCR amplified with the human cDNAs, followed by insertion into psi-CHECK2 vectors using Xhol and NotI (RL-ZEB1 and RL-ZEB2). pCIneo-hRL-ZEB1 WT, pCIneohRL-ZEB1 200b MUT, pCIneo-hRL-ZEB2 WT, and pCIneo-hRL-ZEB2 200b MUT (Gregory et al., 2008; Park et al., 2008) were a gift from G.J. Goodall (University of Adelaide, Adelaide, Australia). pCIneo-hRLZEB1 200 MUT and pCIneo-hRL-ZEB2 200 MUT were generated from pCIneo-hRL-ZEB1 200b MUT and pCIneo-hRL-ZEB2 200b MUT using QuikChange Multi Site-Directed Mutagenesis kit. All primers used for the constructs were shown in Table S3.

The plasmids and/or oligonucleotides were transfected into cells using Lipofectamine 2000 (Invitrogen) or Lipofectamine RNAiMAX (Invitrogen), and incubated for $24-48 \mathrm{~h}$ according to the manufacturer's instructions. Stable cell lines were selected using the appropriate antibiotics for at least $48 \mathrm{~h}$ after transfection.

miRNA microarray analysis. Microarray analysis was performed as described previously (Liu et al., 2004). Differentially expressed miRNAs in HCC cell lines were identified by using Student's $t$ test procedure within class comparison. Differentially expressed miRNAs in HCC tissue samples were also analyzed by class comparison using Student's $t$ test procedure to identify miRNAs up-regulated by WT $\mathrm{p} 53$. The data were deposited in the GEO Expression Omnibus under accession no. GSE2866.

Quantitative real-time (qRT)-PCR and sqRT-PCR. Total RNA was prepared from cells using TRIZOL (Invitrogen) in accordance with manufacturer's instructions. Total RNA was subjected to qRT-PCR or sqRT-PCR Mature miRNAs and other mRNAs were analyzed using the TaqMan miRNA Assays and Gene Expression Assays, respectively, in accordance with manufacturer's instructions (Applied Biosystems). All RT reactions, including no-template controls and RT minus controls, were run in a GeneAmp PCR 9700 Thermocycler (Applied Biosystems). RNA concentrations were determined with a NanoDrop (NanoDrop Technologies, Inc.). Samples were normalized to RNU44 or RNU48 for miRNAs and GAPDH or $\beta$-actin for mRNAs (Applied Biosystems). Gene expression levels were quantified using the ABI Prism 7900HT Sequence detection system (Applied Biosystems). Comparative real-time PCR was performed in triplicate, including no-template controls. Relative expression was calculated using the comparative $\mathrm{Ct}$ method. Probes used for Taqman Assays were shown in Table S4.

For sqRT-PCR, total RNA was reverse-transcribed using the ThermoScript RT-PCR System (Invitrogen) according to the manufacturer's instructions. sqRT-PCR was performed using the primers indicated in Table S3.

Western blot and antibodies. Total cell extracts prepared from cells using RIPA buffer (Sigma-Aldrich) were resolved on $4-20 \%$ gradient SDSpolyacrylamide gel and transferred to PVDF membranes (Bio-Rad Laboratories). Membranes were blocked with blocking solution (5\% skim milk in TBST) and incubated with primary antibody, followed by the incubation with appropriate HRP-conjugated secondary antibody. The primary antibodies used in this study were as follows: anti-p53, anti-CDKN1A, anti- $\beta$-actin, anti-GAPDH, anti-HA-tag, anti-CDH1 (E-cadherin), anti-CDH2 (N-cadherin), anti-VIM (vimentin), anti-ZEB1, and anti-SNAI2 (all from Santa Cruz Biotechnology, Inc.); anti-SNAI1 (Abcam); and anti-ZEB2 (Novus Biologicals). The densitometry of Western blot results was measured using ImageJ software.

p53BS prediction and ChIP assay. Putative p53BSs were predicted by MatInspector (Cartharius et al., 2005; Genomatix).The DNA from C3A cells was prepared and digested with EZ-Zyme Chromatin Prep kit (Millipore). The digested sample was subjected to ChIP assay using EZ-Magna ChIP kit (Millipore). All procedures were done as per the manufacturer's instructions. Anti-p53 (DO-1; Santa Cruz Biotechnology, Inc.) was used for the assay. After ChIP assay was performed, the DNA sample was amplified by PCR using the primers listed in Table S3.

Luciferase assay. Cells were plated in 24 - or 48 -well plates $24 \mathrm{~h}$ before transfection. All plasmids constructed with pGL4-luc2 vectors were cotransfected with control Renilla luciferase plasmid (pGL4-hRluc/TK). pCIneo-hRL-ZEB constructs were cotransfected with control pGL3 vectors (firefly luciferase vector).The ratio of experimental plasmid to control plasmid was 10:1. psiCHECK2 vectors with 3' UTR of ZEB1 or ZEB2 were transfected in a similar manner. Luciferase assays were performed using the Dual-Luciferase Reporter Assay System (Promega). In brief, 24-48 h after transfection, cell lysates were prepared by incubating with $1 \times$ passive lysis buffer for $15 \mathrm{~min}$ at room temperature. Cell lysates were transferred in triplicate to 96-well plates and analyzed using GloMax Luminometer (Promega) according to the manufacturer's instructions.

Immunocytochemistry. Cells grown in chamber slides (Laboratory-Tek) were washed three times with PBS and fixed with 4\% formaldehyde for $15 \mathrm{~min}$ at room temperature. After three PBS washes, cells were permeabilized with $0.25 \%$ Triton X-100 in PBS. Cells were incubated in PBS containing 2\% BSA, followed by overnight incubation at $4^{\circ} \mathrm{C}$ with VIM antibody. The secondary antibody was incubated for $1 \mathrm{~h}$ at room temperature.

Statistics. All graph values represent means \pm SEM from three independent experiments with each measured in triplicate. The differences between two groups were analyzed with unpaired two-tailed Student's $t$ test. $\mathrm{P}<0.05$ was considered statistically significant and indicated with asterisks as described in figure legends.

Online supplemental material. Fig. S1 shows that known EMT-activators other than ZEB1 and ZEB2 are not involved in p53-suppressed EMT. Fig. S2 shows p53-repressed ZEB1/2 expression in p53-null Hep3B cells and the effect of either ZEB1 or ZEB2 knock-down in p53-suppressed EMT. Fig. S3 shows that p53-repressed ZEB1 and ZEB2 expression occurs in the mRNA or translational level, but not in the transcriptional or posttranslational level. Fig. S4 shows the schematics of luciferase constructs used for luciferase activities of ZEB1 or ZEB2 3' UTR. Fig. S5 shows p53-mediated regulation of miR-200 and miR-192 family members and the regulation of ZEB1/2 and EMT markers (CDH1/VIM) through the miRNAs. Table S1 shows the list of p53-regulated miRNAs identified in primary HCC samples. Table S2 shows the list of p53-regulated miRNAs identified in nine HCC cell lines. Table S3 shows information of primers. Table S4 shows information of probes used for qRT-PCR. Online supplemental material is available at http://www.jem.org/cgi/content/full/jem.20110235/DC1.

We thank Dr. B. Vogelstein for $\mathrm{p} 53^{+/+}$and $\mathrm{p} 53^{-1-}$ RKO cells, Dr. Gustavo Leone for p53+t+ and p53-l- MEFs, Dr. Gregory J. Goodall for pClneo-hRL-ZEB1/2 constructs, and Dr. Anders H. Lund for pcDNA3-Zeb2-HA constructs. We also thank Dr. Kay Huebner (The Ohio State University), Dr. Thomas D. Schmittgen (The Ohio State University), and Susan Lutz for careful editing of this paper, and Sharon Palko and Dorothee Wernicke-Jameson for the administrative support.

This work was funded by National Cancer Institute grant RC2 CA148302.

The authors declare no competing financial interests.

Submitted: 31 January 2011

Accepted: 14 April 2011

\section{REFERENCES}

Bartel, D.P. 2004. MicroRNAs: genomics, biogenesis, mechanism, and function. Cell. 116:281-297. doi:10.1016/S0092-8674(04)00045-5

Bracken, C.P., P.A. Gregory, N. Kolesnikoff, A.G. Bert, J. Wang, M.F. Shannon, and G.J. Goodall. 2008. A double-negative feedback loop between ZEB1SIP1 and the microRNA-200 family regulates epithelial-mesenchymal transition. Cancer Res. 68:7846-7854. doi:10.1158/0008-5472.CAN-08-1942 
Braun, C.J., X. Zhang, I. Savelyeva, S. Wolff, U.M. Moll, T. Schepeler, T.F Ørntoft, C.L.Andersen, and M. Dobbelstein. 2008. p53-Responsive micrornas 192 and 215 are capable of inducing cell cycle arrest. Cancer Res. 68:10094-10104. doi:10.1158/0008-5472.CAN-08-1569

Burk, U., J. Schubert, U. Wellner, O. Schmalhofer, E.Vincan, S. Spaderna, and T. Brabletz. 2008. A reciprocal repression between ZEB1 and members of the miR-200 family promotes EMT and invasion in cancer cells. EMBO Rep. 9:582-589. doi:10.1038/embor.2008.74

Calin, G.A., and C.M. Croce. 2006. MicroRNA signatures in human cancers. Nat. Rev. Cancer. 6:857-866. doi:10.1038/nrc1997

Cartharius, K., K. Frech, K. Grote, B. Klocke, M. Haltmeier, A. Klingenhoff, M. Frisch, M. Bayerlein, and T. Werner. 2005. MatInspector and beyond: promoter analysis based on transcription factor binding sites. Bioinformatics. 21:2933-2942. doi:10.1093/bioinformatics/bti473

Chang, T.C., E.A. Wentzel, O.A. Kent, K. Ramachandran, M. Mullendore, K.H. Lee, G. Feldmann, M.Yamakuchi, M. Ferlito, C.J. Lowenstein, et al. 2007. Transactivation of miR-34a by p53 broadly influences gene expression and promotes apoptosis. Mol. Cell. 26:745-752. doi:10.1016/ j.molcel.2007.05.010

Chang, C.J., C.H. Chao, W. Xia, J.Y.Yang, Y. Xiong, C.W. Li, W.H.Yu, S.K Rehman, J.L. Hsu, H.H. Lee, et al. 2011. p53 regulates epithelialmesenchymal transition and stem cell properties through modulating miRNAs. Nat. Cell Biol. 13:317-323. doi:10.1038/ncb2173

Chari, N.S., N.L. Pinaire, L. Thorpe, L.J. Medeiros, M.J. Routbort, and T.J McDonnell. 2009. The p53 tumor suppressor network in cancer and the therapeutic modulation of cell death. Apoptosis. 14:336-347. doi:10 1007/s10495-009-0327-9

Chen,Y.W., D.S. Klimstra, M.E. Mongeau, J.L.Tatem,V. Boyartchuk, and B.C. Lewis. 2007. Loss of p53 and Ink4a/Arf cooperate in a cell autonomous fashion to induce metastasis of hepatocellular carcinoma cells. Cancer Res. 67:7589-7596. doi:10.1158/0008-5472.CAN-07-0381

Georges, S.A., M.C. Biery, S.Y. Kim, J.M. Schelter, J. Guo, A.N. Chang, A.L. Jackson, M.O. Carleton, P.S. Linsley, M.A. Cleary, and B.N. Chau. 2008. Coordinated regulation of cell cycle transcripts by p53-Inducible microRNAs, miR-192 and miR-215. Cancer Res. 68:10105-10112. doi:10.1158/0008-5472.CAN-08-1846

Gregory, P.A., A.G. Bert, E.L. Paterson, S.C. Barry, A. Tsykin, G. Farshid, M.A.Vadas, Y. Khew-Goodall, and G.J. Goodall. 2008. The miR-200 family and miR-205 regulate epithelial to mesenchymal transition by targeting ZEB1 and SIP1. Nat. Cell Biol. 10:593-601. doi:10 $.1038 / \mathrm{ncb} 1722$

Hansen, J.E., L.K. Fischer, G. Chan, S.S. Chang, S.W. Baldwin, R.J. Aragon, J.J. Carter, M. Lilly, R.N. Nishimura, R.H. Weisbart, and M.E. Reeves. 2007. Antibody-mediated p53 protein therapy prevents liver metastasis in vivo. Cancer Res. 67:1769-1774. doi:10.1158/0008-5472.CAN06-3783

He, L., and G.J. Hannon. 2004. MicroRNAs: small RNAs with a big role in gene regulation. Nat. Rev. Genet. 5:522-531. doi:10.1038/nrg1379

He, L., X. He, L.P. Lim, E. de Stanchina, Z. Xuan, Y. Liang, W. Xue, L. Zender, J. Magnus, D. Ridzon, et al. 2007. A microRNA component of the p53 tumour suppressor network. Nature. 447:1130-1134. doi:10 .1038 /nature05939
Hermeking, H. 2007. p53 enters the microRNA world. Cancer Cell. 12:414418. doi:10.1016/j.ccr.2007.10.028

Kato, M., J. Zhang, M. Wang, L. Lanting, H.Yuan, J.J. Rossi, and R. Natarajan. 2007. MicroRNA-192 in diabetic kidney glomeruli and its function in TGF-beta-induced collagen expression via inhibition of E-box repressors. Proc. Natl. Acad. Sci. USA. 104:3432-3437. doi:10.1073/pnas .0611192104

Lewis, B.C., D.S. Klimstra, N.D. Socci, S. Xu, J.A. Koutcher, and H.E.Varmus. 2005. The absence of $\mathrm{p} 53$ promotes metastasis in a novel somatic mouse model for hepatocellular carcinoma. Mol. Cell. Biol. 25:1228-1237. doi:10.1128/MCB.25.4.1228-1237.2005

Liu, C.G., G.A. Calin, B. Meloon, N. Gamliel, C. Sevignani, M. Ferracin, C.D. Dumitru, M. Shimizu, S. Zupo, M. Dono, et al. 2004. An oligonucleotide microchip for genome-wide microRNA profiling in human and mouse tissues. Proc. Natl. Acad. Sci. USA. 101:9740-9744. doi:10 $.1073 /$ pnas.0403293101

Park, S.M., A.B. Gaur, E. Lengyel, and M.E. Peter. 2008. The miR-200 family determines the epithelial phenotype of cancer cells by targeting the E-cadherin repressors ZEB1 and ZEB2. Genes Dev. 22:894-907. doi:10 $.1101 /$ gad.1640608

Pichiorri, F., S.S. Suh, A. Rocci, L. De Luca, C. Taccioli, R. Santhanam, W. Zhou, D.M. Benson Jr., C. Hofmainster, H. Alder, et al. 2010. Downregulation of p53-inducible microRNAs 192, 194, and 215 impairs the p53/MDM2 autoregulatory loop in multiple myeloma development. Cancer Cell. 18:367-381. doi:10.1016/j.ccr.2010.09.005

Raver-Shapira, N., E. Marciano, E. Meiri, Y. Spector, N. Rosenfeld, N. Moskovits, Z. Bentwich, and M. Oren. 2007. Transcriptional activation of miR-34a contributes to p53-mediated apoptosis. Mol. Cell. 26:731743. doi:10.1016/j.molcel.2007.05.017

Ridgeway, A.G., J. McMenamin, and P. Leder. 2006. P53 levels determine outcome during beta-catenin tumor initiation and metastasis in the mammary gland and male germ cells. Oncogene. 25:3518-3527. doi:10.1038/ sj.onc.1209391

Soussi, T. 2007. p53 alterations in human cancer: more questions than answers. Oncogene. 26:2145-2156. doi:10.1038/sj.onc.1210280

Thiery, J.P., and J.P. Sleeman. 2006. Complex networks orchestrate epithelialmesenchymal transitions. Nat. Rev. Mol. Cell Biol. 7:131-142. doi:10 $.1038 / \mathrm{nrm} 1835$

Vassilev, L.T., B.T.Vu, B. Graves, D. Carvajal, F. Podlaski, Z. Filipovic, N. Kong, U. Kammlott, C. Lukacs, C. Klein, et al. 2004. In vivo activation of the p53 pathway by small-molecule antagonists of MDM2. Science. 303:844848. doi:10.1126/science.1092472

Vogelstein, B., D. Lane, and A.J. Levine. 2000. Surfing the p53 network. Nature. 408:307-310. doi:10.1038/35042675

Wellner, U., J. Schubert, U.C. Burk, O. Schmalhofer, F. Zhu, A. Sonntag, B. Waldvogel, C. Vannier, D. Darling, A. zur Hausen, et al. 2009. The EMT-activator ZEB1 promotes tumorigenicity by repressing stemnessinhibiting microRNAs. Nat. Cell Biol. 11:1487-1495. doi:10.1038/ ncb1998

Yang, J., and R.A. Weinberg. 2008. Epithelial-mesenchymal transition: at the crossroads of development and tumor metastasis. Dev. Cell. 14:818-829. doi:10.1016/j.devcel.2008.05.009 